Meta

Journal des traducteurs

Translators' Journal

\title{
L'interprétation de conférence et la connaissance des langues : quelques réflexions
}

\section{Daniel Gile}

Volume 30, numéro 4, décembre 1985

URI : https://id.erudit.org/iderudit/002896ar

DOI : https://doi.org/10.7202/002896ar

Aller au sommaire du numéro

Éditeur(s)

Les Presses de l'Université de Montréal

ISSN

0026-0452 (imprimé)

1492-1421 (numérique)

Découvrir la revue

Citer cet article

Gile, D. (1985). L'interprétation de conférence et la connaissance des langues : quelques réflexions. Meta, 30(4), 320-331. https://doi.org/10.7202/002896ar d'utilisation que vous pouvez consulter en ligne.

https://apropos.erudit.org/fr/usagers/politique-dutilisation/ 


\section{L'INTERPRÉTATION DE CONFÉRENCE ET LA CONNAISSANCE DES LANGUES : QUELQUES RÉFLEXIONS}

DANIEL GILE

\section{INTRODUCTION}

Contrairement à une idée répandue, les interprètes de conférence ne sont pas toujours polyglottes; en fait, ils ont rarement plus de trois ou quatre langues de travail, dont une ou deux langues actives et une ou deux langues passives (voir plus loin). Ce bagage linguistique peut paraître quantitativement modeste, mais les exigences sont strictes sur le plan qualitatif. Pour D. Seleskovitch, directeur de l'École supérieure d'interprètes et de traducteurs de Paris (Ésit), les langues de travail " doivent être connues à la perfection, avec notamment, en ce qui concerne les langues étrangères, une perception pratiquement équivalente à celle d'un individu pour qui elles sont des langues maternelles" (1968: 134).

La sévérité de ces exigences peut surprendre si l'on pense qu'à tout moment, une communication opérationnelle s'établit entre des interlocuteurs de tous pays utilisant des langues qu'ils maîtrisent imparfaitement pour échanger des informations complexes dans des domaines spécialisés (voir Seleskovitch 1981 : 35). Dans ces conditions, on ne comprend pas pourquoi les connaissances linguistiques demandées aux interprètes se situent à un niveau si différent.

Le présent article explique les besoins linguistiques des interprètes sous l'éclairage d'une analyse des contraintes et des efforts ; il évoque les interventions pédagogiques consacrées par les interprètes-enseignants au perfectionnement linguistique des étudiants, et présente des perspectives de recherche dans l'optique d'une éventuelle amélioration de la qualité du travail d'interprétation.

\section{LES LANGUES DE TRAVAIL : LANGUES ACTIVES ET LANGUES PASSIVES}

En sa double qualité de récepteur et d'émetteur, l'interprète a une ou plusieurs langues passives, à partir desquelles il interprète vers sa ou ses langues actives.

L'AIIC (Association internationale des interprètes de conférence) distingue deux types de langues actives :

- les langues « $\mathrm{A}$ ", langues maternelles ou rigoureusement équivalentes à des langues maternelles;

- les langues « $\mathbf{B} »$ qui, sans être des langues maternelles, permettent aux interprètes de se faire comprendre parfaitement.

- Quant aux langues passives, les langues " C ", l'interprète doit en avoir une " compréhension totale» (AIIC $1982: 10$ ).

La plupart des membres de l'AIIC ont une combinaison linguistique trilingue $A B C$. Certains ont plusieurs « $B$ » ou « $C$ », d'autres n'ont pas de langue « $B$ » ou de langue "C ». Les " double $A$ ", dit " bilingues vrais » (Thiéry 1975), sont peu nombreux, surtout parmi les jeunes interprètes, car les conditions susceptibles d'engendrer cette combinaison exceptionnelle sont devenues rares. 
La classification des langues de travail en " $\mathbf{A}$ », « $\mathbf{B}$ » et « $\mathbf{C}$ » soulève depuis de nombreuses années des controverses parmi les membres de l'AIIC ; les désaccords sont imputables à une certaine inadéquation des définitions théoriques à une réalité complexe, fluctuante et difficilement mesurable, ainsi qu'à l'absence de données précises et fiables sur les liens entre le profil de la compétence linguistique de l'interprète et la qualité de son travail (voir Gile : " Aspects méthodologiques de l'évaluation de la qualité du travail en interprétation simultanée ", META, 28-3). Néanmoins, aucune solution de remplacement acceptable n'a été trouvée jusquà présent (voir par exemple Reinert 1976 et Keiser $1978: 15$ ).

\section{LES HANDICAPS DE L'INTERPRÈTE EN TANT QU'AUDITEUR}

Avant même qu'interviennent les contraintes techniques de l'interprétation, les conditions de réception du message sont sensiblement plus difficilles pour l'interprète que pour le destinataire du discours.

\section{L'intégrité du message}

L'auditeur-destinataire (le délégué) retient la partie du discours qui attire son attention; il peut se dispenser de se concentrer sur :

- les informations non pertinentes (formules de politesse, digressions, autres éléments qui ne l'intéressent pas) ;

- les informations redondantes (déjà connues de l'auditeur ou acquises pendant le discours).

L'interprète, pour sa part, est tenu de restituer l'ensemble des informations véhiculées par le discours original, même s'il les juge non pertinentes. Son infériorité cognitive par rapport à l'orateur et aux destinataires (Seleskovitch 1968, Moskowitz 1973, Déjean Le Féal 1978, Lederer 1978) l'empêche d'ailleurs d'identifier avec certitude les éléments qui, pour les destinataires, sont dépourvus d'intérêt ou redondants.

\section{La compensation extra-linguistique}

Le bagage cognitif de l'auditeur — connaissance du sujet, de l'orateur, des intérêts en jeu - lui permet de rétablir le sens d'un énoncé qui dépasse ses connaissances linguistiques (d'ailleurs, sa maîtrise du technolecte de la conférence est généralement supérieure à sa compréhension globale de la langue concernée, s'il s'agit d'une langue étrangère).

L'interprète, profane, ne peut compter sur son bagage cognitif de la même manière. Bien au contraire, il est souvent amené à mobiliser ses connaissances linguistiques pour faire l'analyse d'une idée qui lui est peu familière ou qui lui paraît peu claire : " dès qu'il se sent trahi par ses connaissances de généraliste, il s'accroche à la langue, sa seule spécialité " (Ilg $1980: 119$ — voir aussi Seleskovitch $1975:$ 50-52).

\section{L'intensité de l'effort}

L'auditeur-destinataire se concentre sur l'assimilation des informations pertinentes et peut laisser son intention se relâcher dans les intervalles. Son activité mentale au cours de la réception présente une alternance entre la concentration et le repos.

Les périodes de repos de l'interprète, qui doit assimiler une quantité d'informations nouvelles plus importante, sont moins fréquentes et plus courtes. En outre, l'attention de l'interprète est davantage sollicitée par l'analyse du discours (fond et forme), qui lui est peu familier. Ces deux éléments se conjuguent pour lui demander un effort de concentration considérable - celui de tout profane qui essaie de suivre un discours spécialisé s'adressant à des initiés (rappelons que ces observations ne tiennent pas compte 
des contraintes techniques inhérentes à l'interprétation proprement dite, qui décuplent la difficulté).

\section{LA CONNAISSANCE DES LANGUES DE TRAVAIL : LES BESOINS}

Les chercheurs sont unanimes à affirmer qu'une interprétation de qualité ne peut s'identifier à une succession de réflexes linguistiques, et qu'elle comporte obligatoirement des efforts conscients et dirigés. La qualité de ces efforts détermine en grande partie la qualité de la prestation. C'est pourquoi il semble utile d'aborder des besoins linguistiques des interprètes sous l'angle des modèles d'efforts.

\section{L'interprétation consécutive}

Dans la consécutive, l'interprète écoute un segment de discours de quelques minutes (rarement plus d'un quart d'heure), puis il restitue le message dans la langue d'arrivée. Il doit donc retenir une certaine quantité d'informations pendant un temps relativement long. Il n'y a pas mémorisation des mots, mais analyse du discours à l'aide de techniques précises (Seleskovitch $1968: 93-105$ et Déjean Le Féal $1981: 80-81$ ) et prise de notes, celles-ci ayant un rôle de support (Seleskovitch 1975).

Or, l'analyse du discours se fait sur des entités linguistiques identifiées, et la reconnaissance des formes linguistiques à partir des sons perçus passe obligatoirement par leur catégorisation selon une structure de réception probabiliste (Studdert-Kennedy 1974). Il est important que la reconnaissance linguistique soit aussi rapide et spontanée que possible, afin que l'interprète puisse se concentrer sur les idées. Cette observation justifie la sévérité des exigences formulées par D. Seleskovitch (voir plus haut).

Les contraintes s'exerçant au moment de la restitution ne sont pas identiques aux contraintes s'appliquant à l'écoute et l'analyse ; en effet, le discours interprété est énoncé au rythme de l'interprète, qui a par ailleurs la possibilité de choisir ses mots. La consécutive n'impose donc pas des conditions de rapidité et de spontanéité de l'encodage comme elle en impose pour le décodage.

Par contre, l'énonciation du message au cours de la restitution est soumise à une stricte condition de rigueur. Comme le remarque $\mathrm{C}$. Thiéry, directeur de la section interprétation de l'ÉSIT, l'interprète "doit savoir plier son discours à sa pensée sans se laisser dévier, ni même infléchir, par la facilité avec laquelle telle ou telle expression lui viendrait à la bouche » (Thiéry 1981: 109 — voir aussi de Clarens 1973).

La condition n'est pas banale, tant s'en faut; l'observation montre en effet qu'" une conformité imparfaite de la formulation avec le sens que l'on désire exprimer est l'une des défectuosités notables qui sont le propre de la parole spontanée " (Déjean Le Féal 1981 : 84), et la rigueur dans l'expression demande un réel effort.

En termes de connaissances linguistiques, cette condition est mieux satisfaite et l'interprète possède un vaste vocabulaire actif, qui lui permet d'exprimer avec précision et concision toutes les nuances du message.

Il apparaît donc qu'en interprétation consécutive, les contraintes pèsent surtout sur la langue passive, qui doit être décodée de manière spontanée et rapide. Le fait que cette condition ne soit pas applicable à la langue active explique le comportement des interprètes qui acceptent de travailler en consécutive vers leur langue $B$, alors qu'ils refusent de le faire en simultanée.

\section{L'interprétation simultanée} sécutive :

Deux différences fondamentales distinguent l'interprétation simultanée de la con-

- En consécutive, chaque cycle réception-restitution porte sur des segments de discours de plusieurs centaines, voire plusieurs milliers de mots. En simultanée, les cycles corres- 
pondent en gros à des " unités de sens " (Lederer 1978), véhiculées par des segments d'énoncé qui ne totalisent guère plus de dix ou vingt mots.

- En consécutive, les cycles se suivent sans se chevaucher; en simultanée, ils s'interpénètrent : l'interprète écoute et analyse un segment de discours en même temps qu'il en restitue un autre.

En termes d'efforts, cette deuxième différence implique, en simultanée, une répartition de l'attention de l'interprète entre l'écoute et l'analyse, l'effort de production du discours, et l'effort de mémoire, nécessaire à la restructuration du discours quand les règles de distribution de l'information des deux langues en présence divergent (ces trois efforts, leur situation de concurrence et les incidences de ces relations sont exposés plus en détail dans deux articles à paraître, " Des difficultés de la transmission informationnelle en interprétation simultanée ", et "Les noms propres en interprétation simultanée ").

\section{Les langues passives en interprétation simultanée}

En consécutive, l'écoute s'accompagne d'un effort d'analyse devant servir à la mémorisation à long terme, et d'un effort de prise de notes. En simultanée, à quelques exceptions près (les chiffres et les noms propres par exemple), la prise de notes disparaît. L'effort de mémoire à long terme disparaît également, mais il est remplacé par un effort de mémoire à court terme souvent intense et rendu difficile par ses relations de concurrence avec les deux autres efforts simultanés - écoute et analyse d'une part, et production du discours de l'autre.

Par ailleurs, en consécutive, des défaillances momentanées dans l'écoute et l'analyse peuvent être réparées grâce au contexte, puisque l'interprète intervient après que l'orateur a présenté tout un enchaînement d'idées. En simultanée, de telles défaillances lui sont interdites, car il n'a pas une vue d'ensemble au moment de la restitution (Déjean Le Féal $1981: 80$ ), et ne peut se permettre de prendre un retard supérieur à quelques secondes par rapport à l'orateur, sous peine de perdre des informations (voir Kade et Cartellieri $1971: 15$ et Gile : "Des difficultés de la transmission informationnelle en interprétation simultanée »).

Les exigences de spontanéité et de rapidité dans la compréhension de la langue passive sont donc encore plus fortes en simultanée qu'en consécutive (voir aussi Déjean Le Féal $1981: 86$ ).

\section{Les langues actives en interprétation simultanée}

La concurrence entre les trois efforts concerne à l'évidence les langues actives au même titre que les langues passives. En simultanée, l'interprète suit le rythme de l'orateur non seulement dans l'écoute et l'analyse, mais aussi dans la restitution ; c'est pourquoi les conditions de spontanéité et de rapidité qui, en interprétation consécutive, portent sur le seul décodage, s'appliquent également à l'encodage en interprétation simultanée.

D'autre part, pour décharger sa mémoire (Lederer 1978 : 398) et pour éviter d'insécuriser les auditeurs en restant muet trop longtemps, l'interprète est souvent amené à commencer son discours avant d'avoir pleinement assimilé l'idée de l'orateur. Il doit donc prendre un risque et anticiper, ou choisir un début d'énoncé neutre qui lui permette de suivre l'orateur à mesure qu'il assimile le message. "Chaque fois que le sens de l'énoncé se révèle différent de celui supputé par l'interprète au moment où il commençait à en exprimer les premiers éléments, il doit être capable d'imprimer la direction voulue à son énoncé, sans avoir à se reprendre ni à faire violence à la langue " (Déjean Le Féal 1981 : 91). 
Dans l'expérience quotidienne de l'interprète, il s'agit là d'un problème réel et fréquent, étant donné les différences dans les modalités de distribution de l'information dans les langues concernées et les contraintes grammaticales dans les langues d'arrivée (rareté du passif, nécessité de faire des choix masculin/féminin ou singulier/pluriel à un moment précis, etc. - voir Ilg $1978: 86-88$ ).

L'interprète de simultanée doit donc disposer d'un vaste répertoire de mots et de tournures actives lui permettant de faire face à toutes les situations, d'introduire un complément inattendu quand le verbe choisi est intransitif, de trouver un verbe acceptant le passif quand le sujet présumé de la phrase est susceptible de se révéler être un complément d'objet, de transformer un début d'affirmation en une négation, tout en poursuivant l'analyse de la suite du discours et en retenant les éléments devant être restitués avec quelque retard (voir Herbert 1965 et Ilg $1978: 80$ ).

\section{LE LANGAGE DES CONFÉRENCES}

L'interprétation de conférence demande donc à l'interprète la spontanéité et la rapidité de l'encodage et du décodage, ainsi qu'un vaste vocabulaire actif. Toutefois, ces exigences ne s'appliquent pas de manière homogène à tous les registres linguistiques . Les conférences internationales sont un cadre de communication particulier. On y trouve une "langue officielle", qui peut être écrite mais aussi parlée, "la langue du cours magistral, de la plaidoierie, de la conférence et du discours". Il s'agit d'un discours " scriptural oral " qui se caractérise par " des particularités de style, d'allure générale de l'énoncé, plus élaboré, plus guindé, figé » (Mounin 1975, cité par Ilg 1978).

En règle générale, on peut distinguer quatre composants langagiers dans les conférences internationales :

Le langage non spécialisé : il comporte le vocabulaire général et les structures de base employés dans les échanges non techniques, mais aussi dans les discours spécialisés, qui reposent toujours sur une infrastructure syntaxique et lexicale non technique. À notre connaissance, aucune étude d'envergure sur le " langage non spécialisé " des conférences internationales n'a été réalisée jusqu'à présent, mais des analyses ponctuelles effectuées par l'auteur sur des discours enregistrés donnent à penser que le vocabulaire et les structures concernés sont dans l'ensemble quantitativement restreints et qualitativement simples (étude en cours).

Le langage oratoire : à la différence du " langage non spécialisé », omniprésent dans les conférences internationales comme dans la vie quotidienne, le langage oratoire peut avoir une importance considérable dans certaines réunions diplomatiques ou culturelles et être quasiment absent de certaines réunions techniques.

Le langage oratoire comporte un certain nombre d'axes sémantiques (félicitations, salutations, appels, etc.) qui empruntent un vocabulaire et des tournures distincts de ceux du "langage non spécialisé ». "Nous ne disons pas dans la communication de tous les jours ", explique G. Ilg (1978: 78), « je me félicite de, je me réjouis de, je vous sais gré de, j'abonde dans votre sens, j'applaudis à votre initiative. Mais les orateurs, qu'ils soient diplomates, juristes, économistes ou fonctionnaires, se haussant du col, le font ".

Dans le langage oratoire, la forme et le fond sont intimement liés, et la restitution du discours demande un talent spécifique, le talent oratoire, que l'interprète n'a pas l'occasion d'exhiber dans des réunions plus techniques. 
Le langage de la procédure : ce composant langagier a lui aussi une importance très variable, selon le type de conférence. Dans certaines réunions de travail des organisations internationales, dans les assemblées générales, le langage de la procédure est le principal véhicule de la communication ; dans d'autres réunions, notamment techniques, il n'apparaît pratiquement pas.

Le langage de la procédure, ou " terminologie des conférences ", est quantitativement restreint ; il comporte un certain nombre de termes et de tournures plus ou moins figées qui correspondent à des règles de procédure précises et qui se retrouvent dans les principales langues utilisées dans les conférences internationales (voir Haensch 1965).

Les langages techniques : chaque spécialité a son vocabulaire technique, qui compte quelques centaines, quelques milliers ou quelques dizaines de milliers de mots. À l'évidence, il existe des recoupements entre des domaines connexes ou apparentés, mais la terminologie spécifique de chaque spécialité est souvent très importante, et dans un même domaine, il peut exister plusieurs technolectes distincts, les "vocabulaires maison ».

Les langages techniques ont également ceci de particulier qu'ils se renouvellent et s'enrichissent sans cesse ; d'après " Medias et langage ", la langue française s'accroît annuellement d'environ 10000 termes dans les seuls domaines scientifiques et techniques (n' 16, oct./nov. 1982:22). Enfin, les langages techniques ne sont pas interchangeables ; leur apprentissage demande donc l'acquisition d'une importante quantité de connaissances scientifiques et techniques dispersées et superficielles (voir Gile 1980).

\section{LE PERFECTIONNEMENT LINGUISTIQUE}

L'analyse ci-dessus met en valeur l'hétérogénéité des besoins linguistiques en fonction de la conférence, et conduit à une nette distinction entre les conférences techniques et les réunions non spécialisées.

En effet, si le langage général fait obligatoirement partie de toutes les conférences et doit être parfaitement maîtrisé par tous les interprètes, si le langage de la procédure est suffisamment restreint et bien défini pour qu'il ne pose plus de problèmes après une certaine période de "rôdage ", le langage oratoire et les langages techniques correspondent à des types de conférences distincts et font appel à des compétences linguistiques différentes.

Le langage oratoire se caractérise notamment par un grand déséquilibre entre les connaissances actives et les connaissances passives demandées aux interprètes, respectivement dans leurs langues actives et leurs langues passives : la restitution d'un discours d'apparat demande une maitrise bien plus grande du langage oratoire que sa simple assimilation. Toutefois, en raison de la faible densité informationnelle de ce type de discours, la rapidité et la spontanéité de la réception et de l'encodage y sont moins importantes que dans les autres types de discours.

La situation est tout autre dans les discours techniques, dont la densité informationnelle est élevée, et où la rapidité et la spontanéité d'encodage et de décodage sont essentielles (voir Gile : "Les termes techniques en interprétation simultanée »); dans l'interprétation technique, qui demande des connaissances précises et qui s'appuie sur un " langage non spécialisé " assez simple (énoncés de présentation, identités, inégalités, relations de causalité, etc.), la capacité de décodage et la capacité d'encodage demandées sont plus équilibrées.

À la lumière de ces observations, on comprend aisément pourquoi les discours d'apparat sont presque toujours interprétés vers la langue $\mathbf{A}$, alors que les interventions techniques sont souvent interprétées vers la langue $B$. 
À ce stade, il est possible d'énumérer quatre caractéristiques du type de compétence linguistique demandée aux interprètes pour l'exercice de leur profession :

1. L'interprète doit connaître un très vaste vocabulaire qui comprend des composants techniques très spécialisés, mais où certains registres sont quasiment inexistants.

2. La maîtrise demandée porte sur le mot parlé, et non écrit, sur une capacité de réception auditive, et non visuelle.

3. Un grand déséquilibre entre les connaissances passives (capacité de décodage) et les connaissances actives (capacité d'encodage) est admis.

4. La rapidité et la spontanéité du décodage comme de l'encodage (dans les langues actives) sont essentielles.

À l'évidence, ce genre de compétence ne peut être acquis en trois, quatre ou cinq ans d'études de langues de type classique en faculté. D'après D. Seleskovitch, pour parvenir à une connaissance utile des langues de travail, il faut les avoir acquises dans la prime jeunesse, puis dans les écoles ou les universités étrangères (1968 : 133). Il existe pourtant dans la profession des interprètes compétents, de moins en moins rares, qui ont acquis des langues de travail durant leur adolescence, voire à l'âge adulte, et sans " séjours linguistiques » prolongés, sur la seule base d'un important travail personnel.

Néanmoins, il est généralement admis que

...parmi les programmes appliqués par les écoles, ceux qui correspondent le mieux aux besoins des organisations (et des autres utilisateurs des services d'interprétation - N. de l'auteur) ... visent à enseigner les techniques de la traduction et de l'interprétation à des élèves possédant déjà les connaissances linguistiques requises ... l'étudiant-interprète devrait déjà pleinement posséder ses langues au moment où il accède aux cours d'interprétation, la seule chose qui s'y ajoutera, à ce titre, pendant le cours, c'est l'apprentissage de la terminologie et de la phraséologie typique des réunions internationales. (Keiser 1970 - voir aussi Nilski 1967, Geleff 1971, Lederer 1975, Gravier 1978, Seleskovitch 1981.)

La position est nette ; les écoles d'interprétation refusent le rôle d'écoles de langues. Pourtant, on constate, lors de l'examen d'admission, que le niveau linguistique requis n'est que trop rarement atteint chez les candidats, notamment en ce qui concerne les connaissances actives (Carroll $1978: 121$ ). «Les jeunes gens et les jeunes filles qui souhaitent devenir interprètes souffrent souvent ", dit J. de Clarens, ancienne enseignante à l'ÉSIT, " quoique à un degré plus faible que la plupart de leurs congénères, du mal du siècle : la difficulté de dire " (de Clarens 1973 : 125). En fait, même parmi les candidats admis aux écoles, nombreux sont ceux qui échouent aux épreuves du diplôme faute d'avoir acquis une maitrise suffisante des langues de travail (Keiser 1978 : 13 et 18).

Compte tenu de cette situation, on trouve deux démarches distinctes parmi les enseignants de l'interprétation. À l'ÉSIT, par exemple, on pense que les enseignants peuvent "...orienter les étudiants en leur indiquant où leurs efforts seront les plus utiles", les exercices de perfectionnement linguistique devant toutefois se faire en dehors des cours d'interprétation (Seleskovitch $1981: 42$ ). On notera cependant qu'il y a quelques années seulement, l'ÉSIT avait à son programme deux cours spécifiquement consacrés au perfectionnement linguistique. L'un, animé par J. de Clarens, réunissait les étudiants en une variante de la "debating society" anglo-saxonne et leur donnait l'occasion de préparer et de participer à des débats où ils défendaient souvent des points de vue opposés à leurs véritables opinions (voir de Clarens 1973). Le deuxième cours qui, actuellement, est assuré à l'ÉTI de Genève, était le cours de terminologie des conférences de G. Ilg (1978). 
D'autres enseignants pensent qu'il est utile de consacrer des cours spéciaux au perfectionnement linguistique. À l'école de Genève, il existait un cours hebdomadaire d'une heure intitulé "Élocution et vocabulaire " (Ilg $1959: 3$ ), qui a cédé la place à un cours d'expression orale et de procédure parlementaire confié à G. Lafrance (voir Lafrance 1976) ; G. Ilg continue pour sa part d'animer son cours de terminologie des conférences où il privilégie l'expression orale, s'éloignant de la philosophie qui prédomine actuellement au sein du corps enseignant et qui, à son avis, n'accorde pas aux mots les poids qu'ils méritent (Ilg $1980: 118$ et suivantes).

Dans la pratique, les choses sont moins nettement tranchées, ne serait-ce qu'en raison d'un chevauchement partiel obligatoire des exercices de traduction et d'interprétation d'une part, et des exercices d'expression orale de l'autre.

En effet, par définition, tout exercice d'interprétation comporte un temps de restitution du message, donc d'expression, et les enseignants ne manquent pas de commenter et de critiquer la forme du discours de l'étudiant. Ces exercices font donc véritablement partie d'un effort de perfectionnement linguistique.

La traduction à vue, notamment, est considérée par G. Ilg comme "...un exercice proprement irremplaçable pour acquérir de la souplesse, du vocabulaire et pour apprendre à trancher » $(1978: 73)$.

D'autres exercices, plus techniques, favorisent également le renforcement des aptitudes linguistiques des étudiants ; les exercices consacrés à la recherche et à la formulation de l'idée principale d'un passage (exercice d'analyse), les exercices de reconstitution de phrases présentées avec des éléments manquants (entraînement à l'anticipation verbale), les exercices d'entraînement à la rapidité de réaction verbale (enchaînements rapides questions-réponses), les exercices de simultanéité (écoute et comptage à rebours, écoute et lecture, etc.) pratiqués à l'université d'Innsbruck par B. Moser (1978 : 361-364) favorisent tous le développement des aptitudes verbales.

Des exercices spécifiques sont également effectués dans la grande majorité des cours d'interprétation. L'on citera plus particulièrement la paraphrase, d'usage généralisé (voir Ilg 1978, Moser 1978, Lederer 1981); outre ses vertus dans l'optique du perfectionnement linguistique, elle permet aux étudiants d'interpréter des textes écrits, en les " oralisant".

Dans un article fort intéressant sur le perfectionnement linguistique, K. Déjean Le Féal (1976) présente les exercices qu'elle conseille à ses étudiants. Elle préconise notamment la "lecture complète ", qui consiste à lire un texte en répétant chaque phrase après sa lecture sans avoir recours au texte, ainsi que l'" audition complète ", qui comporte la répétition des phrases et tournures entendues dans la langue étrangère (p. 44).

$K$. Déjean Le Féal conseille aussi d'écouter attentivement les reformulations spontanées que provoquent chez les autochtones des énoncés maladroits prononcés par des étrangers (p. 43-45).

Un autre exercice, l' ' inversion du principe de la forme ", consiste à se concentrer sur les "petits mots", notamment les prépositions, plutôt que sur les mots principaux (verbes, substantifs et adjectifs, qui véhiculent la plus grande partie de l'information - p. 45-46).

La répétition mentale, la répétition à voix basse, l'apprentissage par cœur, la formulation mentale dans la langue étrangère de " toutes les idées qui traversent l'esprit ", la recherche systématique d'une formulation adéquate dans la langue maternelle pour des idées lues ou entendues dans une langue étrangère et inversement (p. 46-49) complètent cette batterie d'exercices qui, au prix d'efforts incessants («chaque instant de la journée peut et doit fournir l'occasion de pratiquer un exercice » - p. 51), doit permettre d'une part d'améliorer les connaissances linguistiques dans la langue maternelle et la 
langue étrangère active, et d'autre part de réduire très sensiblement les interférences linguistiques entre elles.

G. Il se sert beaucoup des exercices de paraphrase , avec « interdiction d'employer les mêmes mots, étymologie ou ressemblance ". "On peut ensuite incorporer des variantes oratoires d'après le procédé de la synecdoque, de la métalepse ou de la métonymie, pour aboutir à l'allusion prestigieuse " $(1978: 73)$.

Les exercices de permutation lui permettent d'illustrer " la variété dans l'assemblage des syntagmes, par inversion, transformation active ou passive, double négation supprimée ou rajoutée, télescopage de constituants discontinus ou, au contraire, application d'incisives" (p. 73).

La recherche de synonymes conduit les étudiants à évoquer « des lexèmes plus inclusifs (archilexèmes, mots génériques, Oberbegriff), puis des lexèmes stylistiquement marqués, plus recherchés, suivis enfin des lexèmes spécialisés » (p. 79).

Les variations, les résumés, les expansions, la chasse aux raccourcis, les exercices d'étoffement, enfin la répétition silencieuse font également partie des méthodes utilisées par G. Ilg à l'école de Genève pour " recenser, exercer, assouplir et enrichir les ressources de chacun" (Ilg $1978: 76$ ).

Dans l'ensemble, le schéma fondamental de la formation linguistique de l'interprète semble toutefois fondé sur le même principe dans toutes les écoles : l'acquisition des langues se fait avant l'admission, et l'enrichissement et l'assouplissement intervenant par la suite ne font que se superposer à des connaissances déjà très solides.

\section{PERSPECTIVES DE RECHERCHE}

On le constate en lisant les différentes publications consacrées au perfectionnement linguistique : les idées et les méthodes reposent sur l'expérience, l'intuition, le bon sens. Le système est opérationnel, puisque les écoles forment des interprètes débutants qui trouvent du travail sur le marché de l'interprétation. Il n'est toutefois pas exclu qu'un examen critique de l'ensemble des méthodes utilisées actuellement puisse favoriser l'éclosion d'idées nouvelles et utiles.

\section{Les langues passives}

Le tour d'horizon pourrait commencer par l'évocation des langues passives, rarement présentes dans les débats méthodologiques; l'attention des interprètesenseignants se focalise en effet sur les langues actives, et l'on serait tenté de croire que les langues passives ne posent pas de problèmes. En fait, nombreux sont les étudiants qui redoublent leur première année ou qui échouent au diplôme en raison d'une langue $\mathrm{C}$ insuffisamment sue.

Ce phénomène, on prend rarement le temps de l'analyser en profondeur, et les enseignants se bornent en général à conseiller aux étudiants concernés une lecture plus intensive dans la langue problématique ou des "séjours linguistiques" prolongés.

En fait, compte tenu de la simplicité linguistique relative des discours utilisés dans. les exercices et des connaissances livresques souvent assez poussées des étudiants, on peut se demander si les défaillances constatées ne se situent pas sur le plan de la spontanéité et de la rapidité du décodage à l'audition, plutôt que sur celui de la connaissance (ou de l'ignorance) des éléments constitutifs des énoncés.

L'apprentissage du lexique et des structures du langage structural oral (voir plus haut) se fait en effet principalement par la voie de la lecture et l'écriture, et non pas par la voie orale. Or, la lecture est moins linéaire que l'écoute, et la reconnaissance des formes par la voie auditive est distincte de la reconnaissance visuelle des signes graphiques.

On est donc fondé à croire qu'une orientation des étudiants vers l'oralisation des connaissances linguistiques est susceptible d'accroître l'efficacité de leurs efforts; 
l'écoute de programmes parlés de stations de radiodiffusion étrangères ou l'enregistrement et l'écoute de textes lus, par exemple, seraient des exercices à évaluer dans cette optique.

S'il s'avère que la faiblesse des étudiants dans leur langue $\mathrm{C}$ tient effectivement à une capacité de reconnaissance auditive peu développée plutôt qu'à des connaissances insuffisantes (le phénomène est frappant chez les étudiants du cours d'initiation aux techniques de l'interprétation japonais-français de l'INALCO - voir Gile : 82), il n'est pas impossible que grâce à des exercices appropriés qui devraient être mis au point, l'on arrive à redresser la situation dans le cadre même de l'écoute (il n'existe pas d'autre formation ayant le même but dans les écoles de langues).

\section{Les langues actives}

La focalisation des interventions des enseignants sur les langues actives s'explique dans le cadre d'une analyse des efforts de l'« équilibre d'interprétation ". En effet, on l'a entrevu plus haut, l'interprétation simultanée peut être considérée comme une suite d'états d'équilibre précaire et de déséquilibre entre l'effort d'écoute et d'analyse, l'effort de mémoire et l'effort de production du discours, la fragilité de l'équilibre étant dans une très grande mesure conditionnée par les contraintes de temps.

Or, dans l'ensemble, on peut dire que la difficulté de l'encodage est supérieure à celle du décodage (Seleskovitch $1981: 42$ ), et que le temps nécessaire à la compréhension du discours est très sensiblement inférieur au temps nécessaire à sa production (on peut suivre sans peine un interlocuteur parlant rapidement ou la lecture rapide d'un texte, mais on a du mal à augmenter fortement un débit de parole naturel). Une intervention efficace des enseignants dans le sens de la production du discours peut donc faire gagner davantage de temps, dans l'absolu, qu'une intervention équivalente orientée vers le décodage, et réduire fortement la fréquence des ruptures d'équilibre génératrices d'incidents (voir Gile : "Des difficultés de la transmission informationnelle ".

En fait, l'intérêt des interventions sur les langues actives de l'interprète est considérable, non seulement dans l'optique de sa formation, mais aussi dans celle de l'activité professionnelle subséquente, et ce sur deux plans :

- Rapidité et spontanéité de l'encodage

Pour réussir aux épreuves du diplôme d'interprète, une certaine aptitude à la production du discours dans les langues actives est exigée. Par la suite, dans leur vie professionnelle, les interprètes trébuchent souvent sur des interventions trop rapides ou trọp denses. Un renforcement de la rapidité et de la spontanéité de l'encodage permettrait de reculer les limites du "faisable".

Dans cette optique, une évaluation de chacun des exercices proposés par G. Ilg, par K. Déjean le Féal et par B. Moser, et de l'efficacité d'une méthode préconisée par $\mathbf{R}$. Pinhas, de l'ISIT, qui consiste à donner des " ficelles " aux étudiants plutôt que de leur proposer des exercices méthodiques (voir par exemple Pinhas 1976), ainsi que la conception et la mise au point d'exercices supplémentaires, présentent un intérêt inconstestable.

- Entretien et amélioration de la qualité des langues de travail

Sans entretien, une langue active se détériore rapidement : la passivation progressive de tournures et d'éléments lexicaux actifs l'appauvrissent en la sclérosant, et la présence permanente des autres langues de travail engendre de dangereux phénomènes d'interférence linguistique (Seleskovitch 1968 : 166-170, Thiéry 1975, Déjean Le Féal 1976 : 48-91, Yagil 1979). 
L'activité professionnelle de l'interprète lui donne l'occasion de réactiver ses connaissances dans le cadre particulier des conférences auxquelles il travaille. Cet entraînement suffit-il à entretenir les langues actives, et notamment les langues $\mathrm{B}$, suspectes (Seleskovitch $1968: 163$ et $1981: 43$ ) ou plus que suspectes (Bros-Brann $1976: 17$ ), ou fautil reprendre périodiquement les exercices de réactivation ?

Le dynamisme, l'intelligence et l'expérience des enseignants ont permis de constituer une batterie d'outils qui assurent une formation professionnelle effective aux candidats à l'interprétation de conférence. Nous pensons toutefois qu'à ce stade de maturité de la pédagogie de l'interprétation, le temps est venu d'apporter la contribution des méthodes scientifiques, quantitatives là où il y a lieu, afin de tenter de trier, d'améliorer, de sélectionner, de trancher dans les débats fondamentaux qui s'éternisent, ou au moins d'y apporter des éléments nouveaux.

La quantification est toujours délicate quand elle porte sur une activité humaine dont le profil est flou et varie d'un individu à l'autre et dans le temps. On trouve néanmoins dans l'interprétation des éléments discrets, donc énumérables, qui sont parfois mesurables dans d'autres dimensions (mode et temps de conjugaison, etc.).

Certains outils mis au point pour répondre à d'autres besoins pourraient être utilisés directement dans la recherche en interprétation; tel est le cas de l'" expressional fluency ", qui mesure la capacité de trouver rapidement un énoncé verbal pour exprimer une idée donnée (French, Ekstrom et Price 1963). D'autres méthodes, notamment celles de la psychologie expérimentale et de la sociologie, pourraient être adaptées et adoptées. Enfin, des outils spécifiques peuvent être créés ad hoc (Gile : "Aspects de l'évaluation de la qualité du travail en interprétation simultanée ", META, 28-3).

B. Moser est à notre connaissance la première enseignante qui ait fait appel à la démarche expérimentale au sens scientifique pour évaluer l'efficacité de ses méthodes (Moser 1978). Nous pensons que la multiplication des initiatives de ce type, appuyées par la rigueur, la prudence dans les extrapolations et la connaissance des conditions de travail réelles des interprètes, est susceptible d'apporter une contribution très précieuse à la profession dans l'optique d'une amélioration de la qualité du travail.

\section{BIBLIOGRAPHIE}

AIIC (1982) : Règlement intérieur de l'assemblée, règlement relatif aux admissions et au classement linguistique. Disciplinary Regulations, Financial Regulations, Genève.

BROS-BRAIN, E. (1976) : Critical comments on H.C. Barik's article « Interpreters Talk a Lot, Among Other Things", dans Bulletin de l'AIIC, IV-1, mars.

CARROLL, J.B. (1978) : "Linguistic Abilities in Translators and Interpreters ", dans D. Gerver and H.W. Sinaiko, Language, Interpretation and Communication, New York, Nato Conference Series, p. 119-130.

de CLARENS, J. (1973) : "L'expression ", dans $E L A, \mathrm{n}^{\circ} 12$, octobre-décembre, p. 124-126.

DÉJEAN LE FÉAL, K. (1976) : "Le perfectionnement linguistique ", $E L A, \mathrm{n}^{\circ}$ 24, octobre-décembre, p. 42-51.

DÉJEAN LE FÉAL, K. (1978) : Lectures et improvisations - incidences de la forme de l'énonciation sur la traduction simultanée (français-allemand), thèse de doctorat de $3^{e}$ cycle, Université de la Sorbonne Nouvelle.

DÉJEAN LE FÉAL, K. (1978) : "L'enseignement des méthodes d'interprétation ", dans J. Delisle (réd.), l'Enseignement de l'interprétation et de la traduction. De la théorie à la pédagogie, Ottawa, Éditions de l'Université d'Ottawa, p. 75-98.

FRENCH, J.W., R.B. EKSTROM, L.A. PRICE (1963) : Kit of Reference Tests for Cognitive Factors, Princeton, N.J., Educational Testing Service.

GELEFF, P. (1971) : «De deux mots, il faut choisir ... le meilleur ", META, 16-1,2, p. 69-74.

GILE, D. (1980) : "Textes spécialisés : techniciens ou traducteurs ? ", dans Traduire, 80-4, décembre.

GILE, D. (1982) : "Initiation à l'interprétation consécutive à l'Institut national des langues et civilisations orientales à Paris ", META, 27-3.

GILE, D. : "Des difficultés de la transmission informationnelle en interprétation simultanée ", envoyé à Babel.

GILE, D. (1985) : "Les termes techniques en interprétation simultanée ", META, 30-3. 
GILE, D. : «Les noms propres en interprétation simultanée ", envoyé à Multilingua.

GILE, D. (1983) : "Aspects méthodologiques de l'évaluation de la qualité du travail en interprétation simultanée ", META, 28-3.

GRAVIER, M. (1978) : "Pédagogie de la traduction ", dans Theory and Practice of Translation, Nobel Symposium 39, Stockholm, 1976, edited by Lillebil Grähs, Gustav Korlin, Bertil, Malmberg, ed. Lang, Berne, p. 201-212.

HAENSCH, G. (1965) : Wörterbuch der Internationale Beziehungen und der Politik Systematisch und Alphabetisch. Deutsch, English, Französisch, Spanisch, München, Max Hueber.

HERBERT, J. (1965) : Manuel de l'interprète, $2^{2}$ éd., Genève, Georg.

ILG, G. (1959) : l'Enseignement de l'interprétation à l'École d'interprètes de Genève, Genève, École d'interprètes.

ILG, G. (1978) : « L'apprentissage de l'interprétation simultanée. De l'allemand vers le français », Parallèle, $n^{\circ} 1$, Cahiers de l'ÉTI, Université de Genève.

ILG, G. (1980) : "L'interprétation consécutive : les fondements ", Paralleles, $\mathrm{n}^{\circ} 3$.

KADE. O. et C. CARTELLIERI (1971) : " Some Methodological Aspects of Simultaneous Interpretation ", Babel, XVII:2.

KEISER, W. (1965) : "Admission dans les écoles d'interprétation", dans Colloque sur l'enseignement de l'interprétation, Paris, les 18 et 19 décembre 1965, AIIC, Genève.

KEISER, W. (1970) : " Les écoles d'interprétation et de traduction répondent-elles à ce que la profession et les employeurs en attendent?", dans l'Interprête, $n^{\circ} 4$.

KEISER, W. (1978) : "Selection and Training of Simultaneous Interpreters ", dans D. Gerver and H.W. Sinaiko, Language, Interpretation and Communication, New York, Nato Conference Series.

LAFRANCE, G. (1976) : Description du cours d'expression orale et de procédure parlementaire, documents de l'ÉTI, Genève.

LEDERER, M. (1975) : " Colloque sur les examens d'entrée aux Écoles d'interprètes ", Paris, 19-20 octobre 1974, dans le Bulletin de l'AIIC, III-2.

LEDERER, M. (1978) : la Traduction simultanée : fondements théoriques, thèse d'État, Paris, Université de la Sorbonne Nouvelle.

LEDERER, M. (1981) : "La pédagogie de la traduction simultanée ", dans J. Delisle (réd.), l'Enseignement de l'inteprétation et de la traduction, de la théorie à la pédagogie, Ottawa, Éditions de l'Université d'Ottawa.

Médias et langage, $\mathrm{n}^{\mathrm{0}} 16$, octobre-novembre 1982.

MOSKOWITZ, D. (1973) : "Le traducteur : récepteur et destinataire du message", dans $E L A, \mathrm{n}^{\circ} 12$, octobre-décembre.

MOUNIN, G. (1975) : Linguistique et philosophie, Paris, Presses universitaires de France.

NILSKI, T. (1967) : "Translators and Interpreters", META, 12-2.

PINHAS, R. (1976) : "Petites ficelles syntaxiques et professionnelles à l'usage des cabines françaises paresseuses ou rebelles à l'empirisme ", dans le Bulletin de l'AIIC, IV-2, août.

REINERT, D. (1976) : "Of 'B"s and wasps..." ", dans le Bulletin de l'AIIC, IV-2, août.

SELESKOVITCH, D. (1968) : l'Interprète dans les conférences internationales, Paris, Lettres modernes Minard.

SELESKOVITCH, D. (1975) : Langage, langues et mémoire, Paris, Lettres modernes Minard.

SELESKOVITCH, D. (1981); "L'enseignement de l'interprétation ", dans J. Delisle (réd.), l"Enseignement de l'interprétation et de la traduction, Ottawa, Éditions de l'Université d'Ottawa.

STUDDERT-KENNEDY, M. (1974) : "The Perception of Speech ", dans T. Sebeok, Current Trends in Linguistics, Paris, The Hague, vol. XII.

THIÉRY, C. (1975) : le Bilinguisme chez les interprètes de conférence professionnels, thèse de doctorat de $3^{\mathrm{e}}$ cycle, Paris, Université de la Sorbonne Nouvelle.

THIÉRY, C. (1981) : "L'enseignement de la prise de notes en interprétation consécutive : un faux problème ? ", dans J. Delisle (réd.), l'Enseignement de l'interprétation et de la traduction, Ottawa, Éditions de l'Université d'Ottawa.

YAGIL, D. (1979) : "Bilinguisme, interférences et traducteurs ", dans Traduire, n 98-1. 